\title{
Expression Invariant Features for Face Recognition
}

\author{
Menta Mohit, Neralla Harichandana, Pendem Bhagyasri, P. M. Ashok Kumar
}

\begin{abstract}
Personal Computer sourced Face Recognition has been a sophisticated and well-found technique which is being rationally utilized for most of the authenticated cases. In reality, there is a number of situations where the expressions of the face will be different. We are here able to instinctively detect the five universal expressions: smile, sadness, anger, surprise, neutral by studying face geometry by determining which type of facial expression has been carried out. Using some facial data with variant expressions. We hereby made some experimentations to calculate the accuracies of some machine learning methods by making some changes in the face images such as a change in expressions, which at last needed for training and recognition identifiers. Our objective is to take the features of neutral facial expressions and add them with the other expressive face images like smiling, angry, sadness to improve the accuracy.
\end{abstract}

keywords: Face recognition, CNN, facial expressions.

\section{INTRODUCTION}

A Face Recognition technology is being used to accurately distinguish an individual through a face image or digital image. It is for the most part utilized in security frameworks. The face recognition will directly use the data from different images or the inputs given to it. The first and the foremost part of facial recognition is it distinguishes each and every person's skin color of the face surface, similarly the curves of the eye gap, jawlines, edges of the nose, and etc. It can see the face from various points to recognize. [3]

It is primarily utilized in air terminals where it will perceive the face and we can stay away from some undesirable fear mongers. When compared different biometrics technologies using the unique marks and iris, recognition of the face has various preferences since it is without contacting the individual. Trough images of the faces we can really detect the individual distinguishing identity from a separation without contacting or collaborating with any of them. And furthermore, face acknowledgment is utilized for wrongdoing confinement reason since face pictures that have been recorded and chronicled, with the goal that it will assist us with identifying an individual later. Restorative determination is one of the most significant zones in which picture handling methodology is helpfully applied[2].

Revised Manuscript Received on December 30, 2019.

* Correspondence Author

Dr. P. M. Shok Kumar*, Associate Professor, Department of Computer Science and Engineering, KL University, Andhra Pradesh, India.

N. Harichandana, Student Department of Computer Science and Engineering, (CSE) Koneru Lakshmaiah Education Foundation Vaddeswaram, Guntur District, Andhra Pradesh, India.

M. Mohit, Department of Computer science and Engineering Koneru Lakshmaiah Education Foundation ,Vaddeswaram, Guntur District. Andhra Pradesh, India.

P. Bhagya, Department of Computer Science and Engineering, Koneru Lakshmaiah Educational Foundation, Vaddeswaram, Guntur District, Andhra Pradesh, India.

(C) The Authors. Published by Blue Eyes Intelligence Engineering and Sciences Publication (BEIESP). This is an open access article under the CC BY-NC-ND license (http://creativecommons.org/licenses/by-nc-nd/4.0/)
Picture preparing is a significant stage so as to improve the exactness both for conclusion methodology and for careful activity. The recognition of Human faces is one of the most predominant and carried out endeavors in present social correlation. All things considered, the facial looks are normal and the identifiers for individuals to represent their sentiments and objectives.If the faces in the dataset are reasonable or if they have a particular demeanor from one of a kind face appearance it's tricky or recognizes the face, so we need to see the face in spite of the way that if the apparent face is having the differing appearance. [1]

We have utilized Deep learning techniques like the Convolutional neural system, and we took a dataset from genuine people and prepared them. At that point, we have tried utilizing the diverse datasets to get the exactness. Specifically, we study the pace of the process of recognizing is to detect the different face parts, like mouth, nose, eyes and the chin and other parts. We additionally study the impact of face acknowledgment objective to zoom out of the facial pictures.

This paper is created in an accompanying manner. Area II quickly displays the other related works of face acknowledgment. Segment III talks about the approach actualized. Segment IV contains the execution and results. In Section V execution estimates utilized are clarified. Area VI contains the end. At last, references are spoken to.

\section{RELATED WORK}

Facial Recognition Technology is still in use and it's been a huge success in many control scenarios. It's utilized in numerous expansive zones, including social media, photography, and mainly in security, Organizations give two primary explanations behind utilizing facial acknowledgment innovation: it assists with security, and it makes photograph altering and sharing simpler.

In this research paper, the author's experiments are in light of utilizing the cutting edge convolutional neural network(CNN) based VGG-Face model which is pre-trained by which separates highlights deep learning. Our decision of model is SVM, to utilize it, the number highlights must be decreased. With the goal that we utilized two such examination one is PCA and the other is LDA to check the correctnesses, we have taken three unique pieces for SVM those are linear, poly and RBF.refer to Table 1.

Ali Elmahmudi, Hassan Ugail [3], these writers talked about novel examinations for testing the presence of some deep learning techniques using the partial face images and different controls on facial pictures, for example, turn and zooming, which we use as preparing and confirmation signs. Our appraisals depend after utilizing the forefront convolutional neural system based structure near to the VGG-Face model which is pre-trained through which we concentrate highlights. They have expressed cosine closeness and the vector machines values to get the accuracy rates by using 2 classifiers.

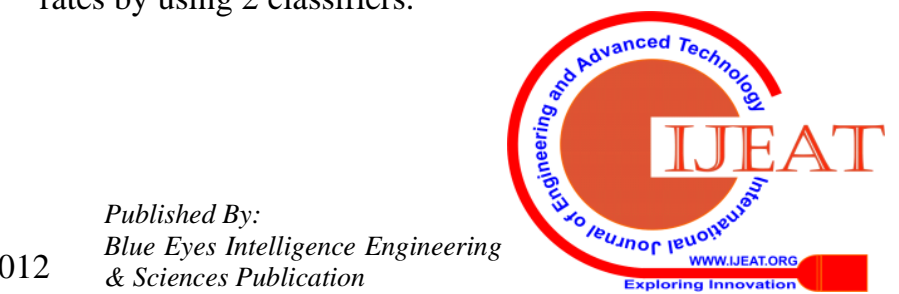


In this examination paper [1]on different FER strategies with three significant advances separately pre-preparing, include extraction and order.

Additionally, the authors showed the advantages of various FER strategies and the presentation experiments of various FER methods. For the most part, FER frameworks meet the issues of variety in enlightenment, present variety, lighting varieties, skin tone varieties.

Omkar M. Parkhi, Andrea Vedaldi, Andrew Zisserman[5], these creators made two commitments: first, they show how an exceptionally enormous scale dataset and talk about the information exchange between virtue and time; second, they have discovered the profound methodologies to recognize the faces accurately to execute the techniques and systems to succeed equivalent class results which are stated best on the standard LFW and YTF face technologies.

Hiroyuki Hase, hogo Tokai, Charoenpong Theekapun [6], these creators contributed by utilizing the face plane calculation, the cross focuses are processed. And afterward, the demeanor face is spoken to as far as uprooting vectors. A removal vector is the development vector of a cross-point pointing from crosswise over purposes of the nonpartisan acknowledgment by methods for help vector machines. The analyses were accomplished for four outward appearances view of the presumption that the individual is known. The outcomes show the achievability of the proposed technique.

\section{PROPOSED METHODOLOGY} Images(dataset collection), Preprocessing the images (data), Extracting features of each data (image), Algorithms and results and are shown in below Fig 1.

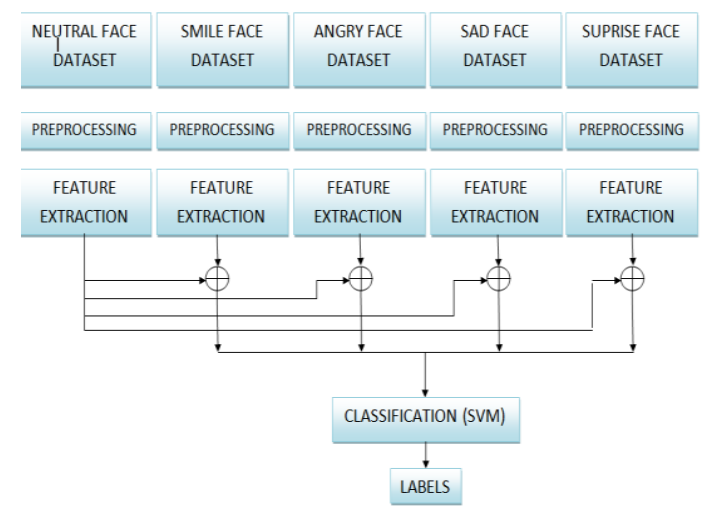

Fig 1. Architecture

\subsection{Collection of the Images:}

We gathered information by capturing genuine faces in our college. And furthermore, some of them are gathered from various online sources. To begin with, we have taken the unbiased essences of every individual then the smiley faces, pitiful faces, irate faces, and shock faces. Along these lines, essentially every individual will have 5 distinct pictures and each is around 20 pictures taken. face to a cross purpose of appearance face. At long last, the relocation vectors are utilized for outward appearance (impartial, anger, surprise and grinning) from 22 people, in

Steps needed in this methodology are collecting the

Thus absolutely they are 70 people that we have taken so every outward appearance dataset that is for unbiased it is 1400 pictures, for smiley additionally 1400 pictures, same with irate, dismal, shock. In this way, aggregate there will be 5 datasets with every outward appearance comprising of 1400 pictures every which in all-out comprises of 7200 pictures.

\subsection{Preprocessing the Images:}

This progression is the essential procedure which will perceive the face in the pictures and concentrates the exact highlights. It incorporates perusing the picture, resizing it and normalizing it.

The following stage will be the element extraction. Allude to fig 1 .

Table 1. Algorithm

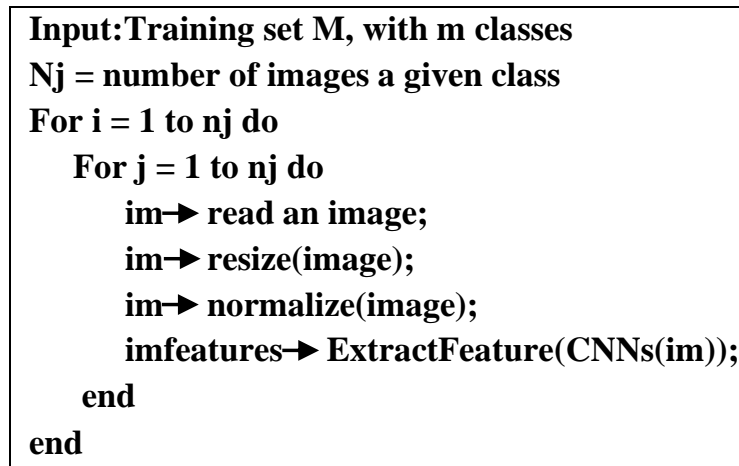

\subsection{Extracting features using VGG:}

There are many pre-trained models for CNN and one of the exceptionally accurate, used in face affirmation is the VGGF model — made by Oxford Visual Geometry Group [5]. The model was set up on a large dataset consisting of 2.6 million face images greater than 2.6 thousand human faces. The designing of VGG includes 38 layers, starts from the data layer up to the yield layer. The data should be a contrasting the image with a size of 224X224, At the point when all is said in done, the VGG constructed for 13 convolutional layers, each layer having an extraordinary game plan of crossbreed parameters. Each of the convolutional layers consists of five pooling layers (max) and there are fifteen linearized straight units (ReLUs). After the above layers, there are 3 totally related layers specifically the Fully connected layers $(6,7,8)$. The last layer is the classifier which is a softmax layer which aggregates the individual face image to classify as a spot. We show the structure of this further in Fig 2.

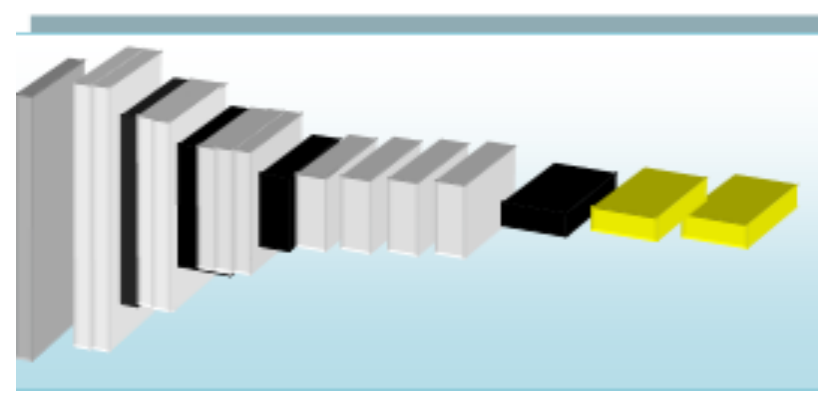

Fig 2. VGG 


\subsection{Classification:}

\subsubsection{PCA and LDA:}

Linear Discriminant Analysis (LDA) is most routinely used as dimensionality decline technique in the pre-getting ready advance for structure course of action and machine learning applications. The goal is to broaden a dataset onto a lowerdimensional space with incredible class-distinctness together decline overfitting ("castigate of dimensionality") and moreover decline computational expenses.

Principal Component Analysis (PCA) is an unsupervised learning technique that is broadly used crosswise over various fields, most noticeably for highlight extraction and dimensionality decrease. Other well-known uses of PCA incorporate exploratory information examinations and denoising of sign in securities exchange exchanging.

\subsubsection{SVM:}

SVM is a managed AI calculation that can be utilized for both double order and multi-grouping issues. The SVM bases on recognizing the edges by methods for hyperplanes to disengage the data into classes.

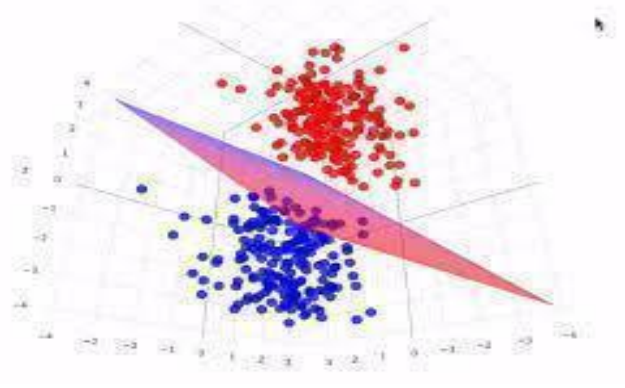

Fig 3. SVM

1. Here we have taken every individual's unbiased face, smiley face, furious face, tragic face, and shock face. In this way, there will be five kinds of datasets, they are nonpartisan, grin, miserable, irate and amazed.

2. At that point, we will have every individual's five diverse outward appearances.

3. Each picture of each dataset will experience preprocessing techniques.

4. At that point, we have to take each picture's component vector utilizing VGG-model. Fig3.1

5. Presently the nonpartisan picture of one individual is included to the component vectors every outward appearance dataset. Along these lines, we get 5 diverse element vectors which are connected to one element vector.

6. Presently we will have a solitary component vector that will be given to PCA and LDA.

7. At that point the names and the preparation set must be given to the SVM with three distinct pieces one is linea, the subsequent one is poly and the third is rbf.

8. So here SVM is utilized for the order.

9. Finally, the yield will be the individual perceived precisely.
10. We need to give any individual's picture with sort of outward appearance so the model that was manufactured ought to remember it precisely.

\section{EXPERIMENTAL RESULTS AND DISCUSSIONS}

A perplexity lattice is the estimation of the viability and execution of our calculation. It is a table with a blend of real perceptions and anticipated perceptions and it is helpful for estimating Accuracy, Precision, Recall, and F1score. We utilized four execution estimates like True Positive (TP), True Negative (TN), False Positive (FP) and False Negative (FN). Genuine Positive methods you foresaw positive and it is genuine True Negative methods have foreseen negative and it is valid. Bogus Positive methods you have foreseen positive and it is bogus. Bogus Negative methods you foreseen negative and it is bogus. Precision, all in all, tells about how normally is our calculation or model is right and it is the extent of accurately evaluated perceptions to the complete perceptions.

Accuracy $=(\underline{\mathrm{TP}}+\mathrm{TN})$

$(\mathrm{TP}+\mathrm{FP}+\mathrm{FN}+\mathrm{TN})$

Precision, tells about when the calculation gauges positive, how regularly it is correct. It is the extent of viably foreseen impression of the all-out foreseen positive recognitions.

Precision $=\quad \underline{T P}$

$$
(\mathrm{TP}+\mathrm{FP})
$$

The recall is likewise called as an affectability which learns what number of the Actual positives our calculation or model catch through stamping it as True Positive

$$
\text { Recall }=\frac{\mathrm{TP}}{(\mathrm{TP}+\mathrm{FN})}
$$

F1 score thinks about both accuracy and review. It is the symphonious mean of accuracy and review.

$$
\text { F1 score }=\frac{2 *(\text { Recall } * \text { Precision })}{(\text { Recall }+ \text { Precision })}
$$

Table 2. The Confusion Matrix of SVM-linear

\begin{tabular}{|l|l|l|l|l|l|}
\hline $\begin{array}{l}\text { Predict } \\
\text { actual }\end{array}$ & $\begin{array}{l}\text { Class } \\
1\end{array}$ & Class 2 & Class 3 & Class 4 & Class 5 \\
\hline Class 1 & 7 & 0 & 0 & 3 & 0 \\
\hline Class 2 & 0 & 4 & 0 & 2 & 0 \\
\hline Class 3 & 0 & 0 & 4 & 2 & 0 \\
\hline Class 4 & 0 & 0 & 0 & 3 & 0 \\
\hline Class 5 & 0 & 0 & 0 & 0 & 5 \\
\hline
\end{tabular}

The model accuracy is 0.76

In this table predicted values and actual values are shown which are predicted using SVM-linear. 
Table 3. The Confusion Matrix of SVM-poly

\begin{tabular}{|c|c|c|c|c|c|}
\hline $\begin{array}{l}\text { Predict } \\
\text { actual }\end{array}$ & $\begin{array}{l}\text { Class } \\
1\end{array}$ & $\begin{array}{l}\text { Class } \\
2\end{array}$ & $\begin{array}{l}\text { Class } \\
3\end{array}$ & $\begin{array}{l}\text { Class } \\
4\end{array}$ & $\begin{array}{l}\text { Class } \\
5\end{array}$ \\
\hline Class 1 & 7 & 0 & 0 & 3 & 0 \\
\hline Class 3 & 0 & 4 & 0 & 2 & 0 \\
\hline Class 4 & 0 & 0 & 4 & 2 & 0 \\
\hline Class 5 & 0 & 0 & 0 & 3 & 0 \\
\hline Class 8 & 0 & 0 & 0 & 0 & 5 \\
\hline
\end{tabular}

The model accuracy is 0.6

In this table predicted values and actual values are shown which are predicted using SVM-poly.

Table 4. The Confusion Matrix of SVM-rbf

\begin{tabular}{|l|l|l|l|l|l|}
\hline $\begin{array}{l}\text { Predict } \\
\text { actual }\end{array}$ & $\begin{array}{l}\text { Class } \\
1\end{array}$ & $\begin{array}{l}\text { Class } \\
2\end{array}$ & $\begin{array}{l}\text { Class } \\
3\end{array}$ & $\begin{array}{l}\text { Class } \\
4\end{array}$ & $\begin{array}{l}\text { Class } \\
5\end{array}$ \\
\hline Class 1 & 7 & 0 & 0 & 3 & 0 \\
\hline Class 2 & 0 & 4 & 0 & 2 & 0 \\
\hline Class 3 & 0 & 0 & 4 & 2 & 0 \\
\hline Class 4 & 0 & 0 & 0 & 3 & 0 \\
\hline Class 5 & 0 & 0 & 0 & 0 & 5 \\
\hline
\end{tabular}

The model accuracy is 0.6

In this table predicted values and actual values are shown which are predicted using SVM-rbf.

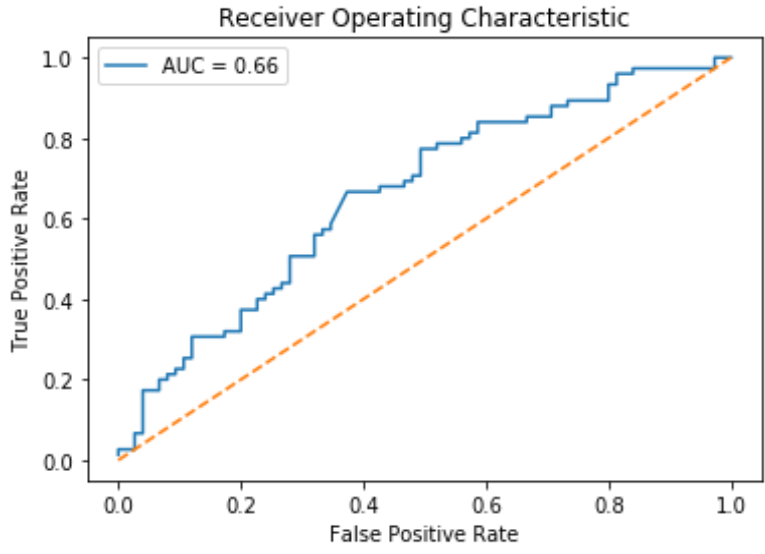

Fig 4. SVM-linear

In this graph the true positive rate and false positive rate is considered and the interval is 0.2.

The accuracy is almost same as compared to SVM-poly and SVM-rbf.

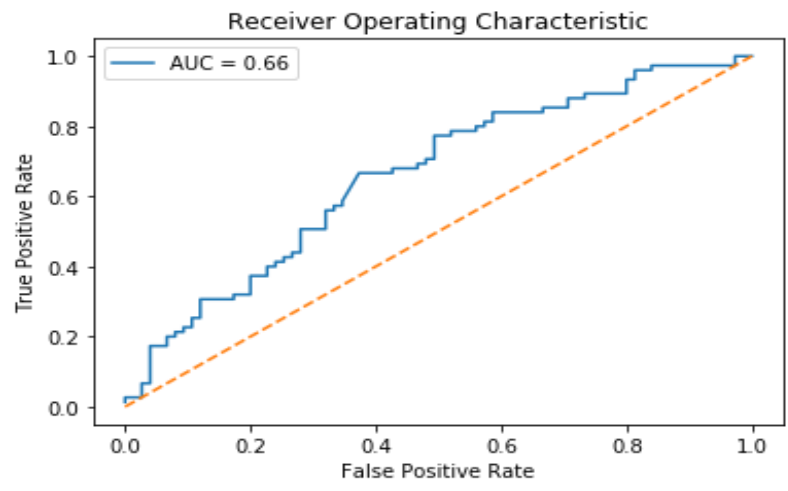

Fig 5. SVM - poly
In this graph the true positive rate and false positive rate is considered and the interval is 0.2. The accuracy is almost same as compared to SVM-rbf and SVM-linear.

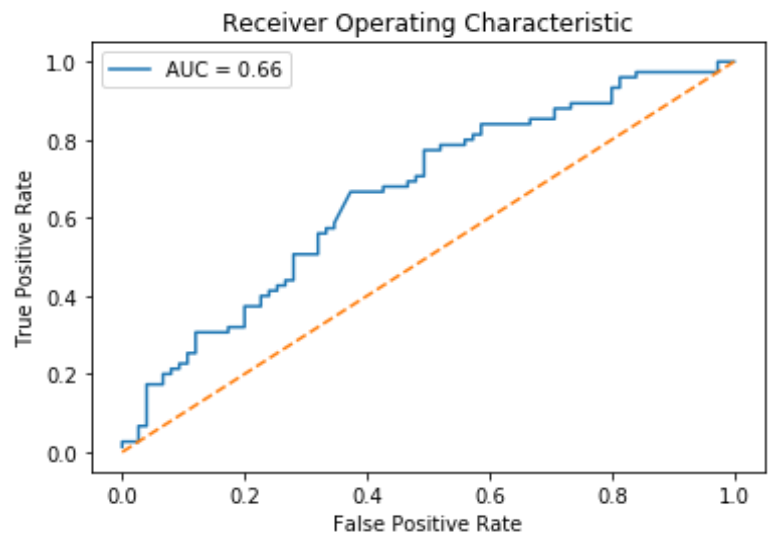

Fig 6. SVM-rbf

In this graph the true positive rate and false positive rate is considered and the interval is 0.2 . The accuracy is almost same as compared to SVM-poly and SVM-linear.

Table 5. Heat map of all the three SVM

\begin{tabular}{|c|c|c|c|c|c|c|}
\hline $\mathbf{0}$ & 7 & 0 & 0 & 3 & 0 & $\mathbf{6 . 0}$ \\
\hline $\mathbf{1}$ & 0 & 4 & 0 & 2 & 0 & $\mathbf{4 . 5}$ \\
\hline $\mathbf{2}$ & 0 & 0 & 4 & 2 & 0 & $\mathbf{3 . 0}$ \\
\hline 3 & 0 & 0 & 0 & 3 & 0 & $\mathbf{1 . 5}$ \\
\hline $\mathbf{4}$ & 0 & 0 & 0 & 0 & 5 & $\mathbf{0 . 0}$ \\
\hline- & $\mathbf{0}$ & $\mathbf{1}$ & $\mathbf{2}$ & $\mathbf{3}$ & $\mathbf{4}$ & - \\
\hline
\end{tabular}

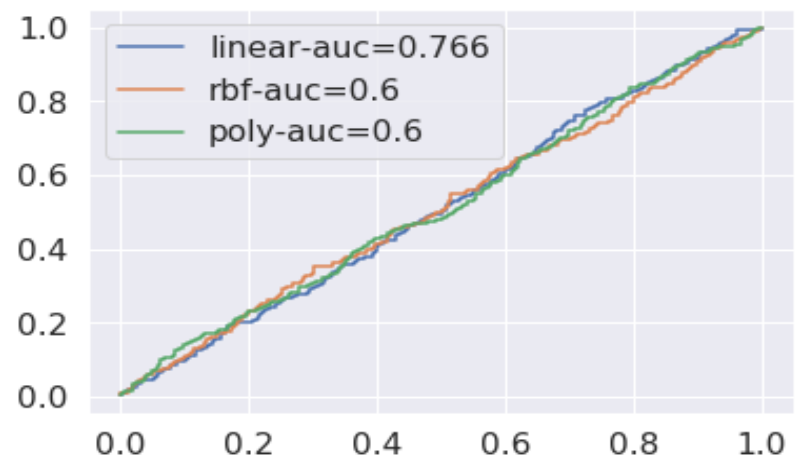

Fig 7. All three kernels graphs

Table 6. Accuracies

\begin{tabular}{|l|l|l|l|}
\hline S.NO & $\begin{array}{l}\text { SVM- } \\
\text { Kernels }\end{array}$ & PCA & LDA \\
\hline 1. & Linear & $76 \%$ & $76.8 \%$ \\
\hline 2. & RBF & $60 \%$ & $60 \%$ \\
\hline 3. & Poly & $60 \%$ & $60.8 \%$ \\
\hline
\end{tabular}




\section{CONCLUSION}

The farthest point with respect to existing machine-based face insistence calculations to perform enough in the events of deficient facial information, for example, blocked faces, turned faces or zoomed out appearances as sign stay a problematic undertaking in the field of PC vision and visual getting ready. In this paper of work, we have demonstrated how an individual is seen if the structure confirmations any of the person's appearance pictures. like on the off chance that we input a furious face of an individual whose mark is one then the framework needs to perceive the name of that individual is one and subtleties of the individual. In the event that we give a pitiful face of an individual whose names must be anticipated as 6 then the framework as to foresee it as the individual with name 6 and his/her subtleties. Consequently, we have fabricated a model that will take any sort of articulation or nonpartisan picture and yield the precise individual with its mark or with his/her subtleties.

\section{REFERENCES}

1. A Survey on Human Face Expression Recognition Techniques I.Michael Revina , W.R. Sam Emmanuel Reg No. 12417, N.M. Christian College, Marthandam Affiliated to Manonmaniam Sunadaranar University, Abishekapatti, Tirunelveli - 627012, Tami Nadu, India Department of Computer Science, N.M. Christian College, Marthandam Affiliated to Manonmaniam Sunadaranar University, Abishekapatti, Tirunelveli - 627012, Tamil Nadu, India

2. Image processing for medical diagnosis using CNN Paolo Arena*, Adriano Basile, Maide Bucolo, Luigi Fortuna Dipartimento Elettrico Elettronico e Sistemistico, Universita Degli Studi di Catania, Viale A. Doria 6, 95125 Catania, Italy.

3. Deep face recognition using imperfect facial data Ali Elmahmudi, Hassan Ugail * Centre for Visual Computing, Faculty of Engineering and Informatics, University of Bradford, Bradford BD7 1DP, UK

4. Rajeev Ranjan, Swami Sankaranarayanan, Carlos D. Castillo and Chellappa, Rama. An all-in-one convolutional neural network for face analysis. In 2017 IEEE 12th International Conference on Automatic Face \& Gesture Recognition. IEEE, 2017.

5. Deep Face Recognition Omkar M. Parkhi omkar@robots.ox.ac.ukAndreaVedaldivedaldi@robots.ox.ac.uk

Andrew Zisserman az@robots.ox.ac.uk Visual Geometry Group Department of Engineering Science University of Oxford

6. Facial Expression Recognition from a Partial Face Image by Using Displacement Vector Charoenpong Theekapun, Shogo Tokai, Hiroyuki Hase Dept. of Information Science, University of Fukui, 39-1 Bunkyo, Fukui 910-8507, Japan E-MAIL: theekapun@gmail.com, tokai@fuis.fuis.fukui-u.ac.jp, hase@fuis.fuis.fukui-u.ac.jp

7. G. B. Huang, M. Ramesh, T. Berg, and E. Learned-Miller. Labeled Faces in the wild: A database for studying face recognition in unconstrained environments. Technical Report 07-49, University of Massachusetts, Amherst, 2007.

8. Wasserstein CNN: Learning Invariant Features for NIR-VIS Face Recognition Ran He, Senior Member, IEEE, Xiang Wu, Zhenan Sun, Member, IEEE, and Tieniu Tan, Fellow, IEEE

9. Th. Charoenpong, T. Shogo and H. Hase, "Facial Expression Recognition from Side View Face by Using Face Plane", Int. Conf. of ICWAPR07, Beijing, pp. 1096-1101, Nov 2007.

10. Liao, Shengcai, et al. "Heterogeneous face recognition from local structures of normalized appearance." International Conference on Biometrics. Springer, Berlin, Heidelberg, 2009.

11. Zhang, Wei, Xiaogang Wang, and Xiaoou Tang. "Coupled information-theoretic encoding for face photo-sketch recognition."Computer Vision and Pattern Recognition (CVPR), 2011 IEEE Conference on. IEEE, 2011.

12. Videla, L.S., Rao, M.R.N., Anand, D., Vankayalapati, H. D., \& Razia, S. (2019). Deformable facial fitting using active appearance model for emotion recognition DOI:10.1007/978-981-13-1921-1_13 Retrieved from www.scopus.com

13. Durga Indira, N., \& Venu Gopala Rao, M. (2018). Automatic facial expression detection system using single face classifier. International
Journal of Engineering and Technology(UAE), 7(3.12 Special Issue 12), 1144-1148. Retrieved from www.scopus.com

14. Vamsi Krishna, M., Bhargav Reddy, A., \& Sandeep, V. (2018). Facial recognition enabled the smart door to unlock the system.International Journal of Engineering and Technology(UAE),7(2),183186,DOI:10.14419/ijet. v7i2.7.10289

15. Sampath Dakshina Murthy, A., Koteswarao Rao, S., Das, R. P., \& Kishore, K. L. (2017). Recognition of facial features by principal component analysis using eigenvalues of variants. Journal of Advanced Research in Dynamical and Control Systems, 9(Special Issue 14), 736-744.

\section{Web references:}

Wikipedia, From Wikipedia, the free encyclopedia, "Convolutional Neural Network"[online], Available https://en.wikipedia.org/wiki/Convolutional_neural_network,[Accessed-on23-October]

\section{AUTHORS PROFILE}

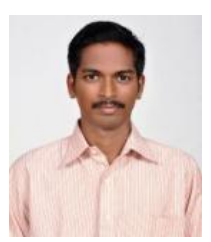

Dr.P.M.Ashok Kumar, obtained his Ph.D. from Anna University, B.Tech in ECE from JNTU Hyderabad and M.E Computer Science Engg. from Anna University, Chennai, India. Currently, he is working as an Associate Professor in the Department of Computer Science Engineering, KL University, India. He authored more than 20 articles in both SCIE \& Scopus indexed journals, conferences. His main research interests include Image processing, Data mining, Machine learning. He is currently working on Face Detection \& Recognition, Video Traffic surveillance, Texture feature extraction, Object tracking features.

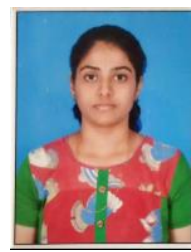

N.Harichandana, is a student of Computer Science And Engineering (CSE) at Koneru Lakshmaiah Education Foundation at Vaddeswaram, Guntur District, Andhra Pradesh

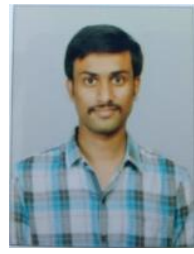

M.Mohit, is a student of the Computer science and Engineering Department at the Koneru Lakshmaiah Education Foundation at Vaddeswaram, Guntur District.

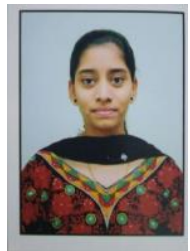

P.Bhagya, Sri is a student of the Computer Science and Engineering Department at the Koneru Lakshmaiah Educational Foundation at Vaddeswaram, Guntur District 